\title{
LIMIT OF A SEQUENCE OF FUNCTIONS WITH ONLY COUNTABLY MANY POINTS OF DISCONTINUITY
}

\section{CHARLES TUCKER}

\section{Introduction and statement of results.}

1.1. Introduction. We present here first an approximation theorem (Theorem 1) for certain limit functions defined on a general topological space. This strengthens a result which may be found in Hausdorff [1]. With the aid of this theorem we characterize the limits of some classes of discontinuous functions in Theorem 2 .

Denote by $S$ a topological space. All functions considered are real valued. Convergence means pointwise convergence unless otherwise stated. Suppose $f$ is a function defined on $S, x \in S$, and $f$ is not continuous at $x$. The statement that $(x, f(x))$ is a removable point of discontinuity means that there exists a function $g$ which agrees with $f$ on $S-\{x\}$ and which is continuous at $x$. The statement that the function $u$ defined on $S$ is upper semicontinuous means that, if $x \in S$ and $d>u(x)$, then there exists a neighborhood $V$ of $x$ such that, if $y \in V$, then $d>u(y)$. The function $l$ is lower semicontinuous if $-l$ is upper semicontinuous.

\subsection{Statement of Theorems.}

Theorem 1. Suppose $M$ is a linear space of real valued functions defined on $S$ which contains a nonzero constant function and which is closed under the operation of absolute value, and $U$ is the set to which $u$ belongs only in case $u$ is the greatest lower bound of a countable subset of $M$. Then, if the function $f$ defined on $S$ is the limit of a sequence of functions in $M$, it is the uniform limit of a sequence each term of which is the difference of two members of $U$, each of which is bounded above.

TheOREм 2. Suppose $S$ is perfectly normal and $f$ is a function defined on $S$. Each two of the following three statements are equivalent:

(1) the function $f$ is the limit of a sequence of functions, each of which has at most a finite number of points of discontinuity, each of which is removable;

(2) the function $f$ is the limit of a sequence of functions, each of which has at most countably many points of discontinuity; and

(3) there exist a function $g$ which is the limit of a sequence of continuous functions and a countable subset $T$ of $S$ such that, if $x \in S-T$, $f(x)=g(x)$.

Received by the editors October 19, 1966. 


\section{Proof of theorems.}

2.1. Proof of Theorem 1. The following facts should be noted: As $M$ is closed under the operation of absolute value, if each of $h$ and $k$ is in $M$ then each of $\max \{h, k\}$ and $\min \{h, k\}$ is in $M$. Also, each member of $U$ is the limit from above of a monotonic sequence of members of $M$.

Suppose $\left\{f_{p}\right\}_{p=1}^{\infty}, f_{p} \in M$, converges to $f$. For each positive integer $p$ define $l_{p}=$ l.u.b. $\left\{f_{p}, f_{p+1}, \cdots\right\}$ and $u_{p}=$ g.l.b. $\left\{f_{p}, f_{p+1}, \cdots\right\}$. Thus $-l_{p} \in U, u_{p} \in U, l_{p} \geqq f_{p} \geqq u_{p}, l_{p+1} \leqq l_{p}$, and $u_{p} \leqq u_{p+1}$. Suppose $c>0$. Define $R_{p}=\left\{x \mid l_{p}(x)-u_{p}(x) \leqq c\right\}$. We assume that $R_{1}$ contains at least one point. Note that $\cup_{p=1}^{\infty} R_{p}=S$.

Now we show that the function $g_{p}$ defined such that $g_{p}(x)=1$ if $x \in R_{p}$ and $g_{p}(x)=0$ if $x \notin R_{p}$ is in $U$. There exists a monotonic sequence $\left\{v_{n}\right\}_{n=1}^{\infty}, v_{n} \in M$, converging from above to $u_{p}$ and there exists a monotonic sequence $\left\{w_{n}\right\}_{n=1}^{\infty}, w_{n} \in M$, converging from below to $l_{p}$. Define $h_{n}=w_{n}-v_{n}$ and $Q_{n}=\left\{x \mid h_{n}(x) \leqq c\right\}$. Thus $R_{p}=\bigcap_{n=1}^{\infty} Q_{n}$. Define $q_{n}(x)=1$ if $x \in Q_{n}$ and $q_{n}(x)=0$ if $x \in Q_{n}$. Define $d_{n}$ $=\max \left\{1-\left(\max \left\{h_{n}, c\right\}-c\right), 0\right\}$. Now $d_{n}(x)=1$ if $x \in Q_{n}$ and $0 \leqq d_{n}(x)<1$ if $x \notin Q_{n}$. Define $r_{n, i}=\max \left\{i \cdot d_{n}-i+1,0\right\}$. Thus $\left\{r_{n, i}\right\}_{i=1}^{\infty}, r_{n, i} \in M$, is a monotonic sequence converging to $q_{n}$ from above. Define $\alpha_{i, p}$ $=\min \left\{r_{1, i}, r_{2, i}, \cdots, r_{i, i}\right\}$. Then it is true that $\left\{\alpha_{i, p}\right\}_{i=1}^{\infty}, \alpha_{i, p} \in M$, is a monotonic sequence converging to $g_{p}$ from above and therefore $g_{p} \in U$.

Define $f_{0}=0$,

$$
s_{p}=\sum_{n=1}^{p} \max \left\{f_{n}-f_{n-1}, 0\right\},
$$

and

$$
t_{p}=\sum_{n=1}^{p} \min \left\{f_{n}-f_{n-1}, 0\right\} .
$$

Note that $s_{p}+t_{p}=f_{p}$. Define $h(x)=s_{p}(x)$ and $k(x)=t_{p}(x)$ if $x \in R_{p}$ but $x \notin R_{p-1}$. Note that both $k$ and $-h$ are bounded above.

Now we show that each of $k$ and $-h$ is in $U$. Define $\beta_{i, p}=i \cdot \alpha_{i, p}-i$. Thus $\beta_{i, p}(x)=0$ for $x \in R_{p}, \beta_{i, p}(x)<0$ for $x \notin R_{p}$, and $\beta_{i, p}(x) \rightarrow-\infty$ as $i \rightarrow \infty$ if $x \notin R_{p}$. Define $\delta_{i, 1}=\max \left\{\beta_{i, 1}+t_{1}, t_{2}\right\}$. Define $\delta_{i, n}$ $=\max \left\{\beta_{i, n}+\delta_{i, n-1}, t_{n+1}\right\}$ if $n>1$. Define $\gamma_{i}=\delta_{i, i}$. If $p \leqq i$ and $x \in R_{p}$, $\gamma_{i}(x)=\delta_{i, p}(x)$. Thus $\left\{\gamma_{i}\right\}_{i=1}^{\infty}, \gamma_{i} \in M$, is a monotonic sequence converging from above to $k$ and $k \in U$. A similar argument shows that $-h$ is in $U$.

If $x \in R_{p}$ but $x \notin R_{p-1}$, then $h(x)=s_{p}(x), k(x)=t_{p}(x)$, and $h(x)$ $+k(x)=f_{p}(x)$. By the way $R_{p}$ was defined 


$$
|f(x)-h(x)-k(x)|=\left|f(x)-f_{p}(x)\right| \leqq c .
$$

2.2. Notation. Suppose $R$ is a subset of $S$. Denote by $U(R), L(R)$, and $C(R)$, respectively, the set of all upper semicontinuous functions, lower semicontinuous functions, and continuous functions defined on $R$. Denote by $C_{1}(R)$ the set of all functions which are the limit of a sequence of members of $C(R)$. Denote by $U(R)+L(R)$ the set $\{f \mid f=h+k, h \in U(R) k \in L(R)\}$.

2.3. Some properties of semicontinuous functions. Certain facts concerning semicontinuous functions should be recalled. Property (1) is that if $\left\{f_{p}\right\}_{p=1}^{\infty}, f_{p} \in C(S)$, converges to $f$, then there exists a sequence $\left\{u_{p}\right\}_{p=1}^{\infty}, u_{p} \in U(S), u_{p+1} \geqq u_{p}$, converging to $f$ and a sequence $\left\{l_{p}\right\}_{p=1}^{\infty}$, $l_{p} \in L(S), l_{p+1} \leqq l_{p}$, converging to $f$ such that $l_{p} \geqq f_{p} \geqq u_{p}$. This can be verified by defining $u_{p}=$ g.l.b. $\left\{f_{n} \mid n=p, p+1, \cdots\right\}$ and $l_{p}=$ 1.u.b. $\left\{f_{n} \mid n=p, p+1, \cdots\right\}$. Property (2) is that $S$ is perfectly normal if and only if every $u \in U(S)$ is the limit from above of a monotonic sequence of continuous functions. This is a theorem due to Hing Tong [2].

2.4 Proof of Theorem 2.

(a) $1 \rightarrow 2$ : This follows immediately.

(b) $2 \rightarrow 3$ : Suppose $\left\{f_{p}\right\}_{p=1}^{\infty}$ is a sequence of functions defined on $S$ converging to $f$ such that each term of the sequence has at most countably many points of discontinuity. Define $T=\left\{x \mid f_{p}\right.$ is discontinuous at $x$ for some positive integer $p\}$. The set $T$ is countable. Define $R=S-T, r_{p}$ to be the restriction of $f_{p}$ to $R$, and $r$ to be the restriction of $f$ to $R$. Thus, $\left\{r_{p}\right\}_{p=1}^{\infty}, r_{p} \in C(R)$, converges to $r$. Now we apply Theorem 1. We take $M$ to be the set of all continuous functions defined on $R$. The limit from above of a monotonic sequence of continuous functions is upper semicontinuous. Therefore, the set $U$ of Theorem 1 is a subset of $U(R)$. Thus, $r$ is the uniform limit of a sequence $\left\{b_{p}\right\}_{p=1}^{\infty}$, where each $b_{p}$ is the difference of two upper semicontinuous functions, each bounded above. A function $u \in U(R)$ which is bounded above can be extended to a function $v \in U(S)$ by defining $v(x)=$ g.l.b. $\{$ l.u.b. $\{u(y) \mid y \in V\} \mid V$ a neighborhood of $x\}$ where this lower bound exists. There exist at most a countable number of points $x_{1}, x_{2}, \cdots$ of $S$ where the lower bound does not exist. At these points define $v\left(x_{p}\right)=-p$. By this means $b_{p}$ can be extended to a function $d_{p} \in U(S)+L(S)$. Since $\left\{d_{p}\right\}_{p=1}^{\infty}$ converges uniformly to $f$ on $R$, we can assume without loss of generality that $\left|d_{p}(x)-f(x)\right|$ $<1 / p$ for $x \in R$ and all $p$. Define $c_{p}=d_{p}+1 / p$ and $e_{p}=d_{p}-1 / p$. If $x \in R$ and $p$ and $j$ are positive integers, $c_{p}(x) \geqq f(x) \geqq e_{j}(x)$. Also, if $x \in R, c_{p}(x) \rightarrow f(x)$ as $p \rightarrow \infty$ and $e_{p}(x) \rightarrow f(x)$ as $p \rightarrow \infty$. 
Define $s_{p}=\min \left\{c_{1}, c_{2}, \cdots, c_{p}\right\}$ and $t_{p}=\max \left\{e_{1}, e_{2}, \cdots, e_{p}\right\}$. If $x \in R, s_{p}(x) \geqq f(x) \geqq t_{p}(x)$. If $x \in S, s_{p}(x) \geqq s_{p+1}(x)$ and $t_{p}(x) \leqq t_{p+1}(x)$. Define $v_{p}=\min \left\{\max \left\{s_{1}, t_{1}\right\}, \max \left\{s_{2}, t_{2}\right\}, \cdots, \max \left\{s_{p}, t_{p}\right\}\right\}$ and $w_{p}=\max \left\{\min \left\{s_{1}, t_{1}\right\}, \min \left\{s_{2}, t_{2}\right\}, \cdots, \min \left\{s_{p}, t_{p}\right\}\right\}$. If $x \in R$, $v_{p}(x) \rightarrow f(x)$ as $p \rightarrow \infty$ and $w_{p}(x) \rightarrow f(x)$ as $p \rightarrow \infty$. If $x \in S, v_{p}(x) \geqq v_{p+1}(x)$ $\geqq w_{p+1}(x) \geqq w_{p}(x)$.

If both $\alpha$ and $\beta$ are in $C_{1}(S)$ then both $\max \{\alpha, \beta\}$ and $\min \{\alpha, \beta\}$ are also in $C_{1}(S)$. From this and property (2) of $\$ 2.3$ it follows that $v_{p}$ and $w_{p}$ are each in $C_{1}(S)$.

By property (1) of $\$ 2.3$, for each positive integer $p$, there exist a sequence $\left\{l_{p, n}\right\}_{n=1}^{\infty}, \quad l_{p, n} \in L(S), \quad l_{p, n} \geqq l_{p, n+1}$, converging to $v_{p}$ on $S$ and a sequence $\left\{u_{p, n}\right\}_{n=1}^{\infty}, u_{p, n} \in U(S), u_{p, n} \leqq u_{p, n+1}$, converging to $w_{p}$ on $S$. Define $m_{p}=\min \left\{l_{1, p}, l_{2, p}, \cdots, l_{p, p}\right\}$ and $q_{p}$ $=\max \left\{u_{1, p}, u_{2, p}, \cdots, u_{p, p}\right\}$. The sequence $\left\{m_{p}\right\}_{p=1}^{\infty}, m_{p} \in L(S)$, $m_{p} \geqq m_{p+1}$, converges to $f$ on $R$. The sequence $\left\{q_{p}\right\}_{p=1}^{\infty}, q_{p} \in U(S)$, $q_{p} \leqq q_{p+1}$, converges to $f$ on $R$. Further, $m_{p} \geqq q_{p}$.

Define $g$ to be the average of the limit of $\left\{m_{p}\right\}_{p=1}^{\infty}$ and the limit of $\left\{q_{p}\right\}_{p=1}^{\infty}$. As both sequences converge to $f$ on $R, g$ agrees with $f$ on $R$. Define $\alpha_{p}=m_{p}+1 / p$ and $\beta_{p}=q_{p}-1 / p$. As $\alpha_{p} \in L(S), \beta_{p} \in U(S)$, and $\alpha_{p}>\beta_{p}$, by a theorem due to Nagami [3] there exists a function $g_{p} \in C(S)$ such that $\alpha_{p}>g_{p}>\beta_{p}$. Denote the points of $T$ as $\left\{x_{1}, x_{2}, \cdots\right\}$. The function $g_{p}$ can be chosen so that it agrees with $g$ at the first $p$ points of $T$. This statement can be justified as follows: Consider the first two points of $T, x_{1}$ and $x_{2}$. If every neighborhood of $x_{1}$ contains $x_{2}$ or vice versa then every continuous function has the same value at $x_{1}$ and $x_{2}$. This is also true of the functions in $C_{1}(S)$, in particular those in $U(S)$ and $L(S)$. Thus, $g\left(x_{1}\right)=g\left(x_{2}\right)$. As $S$ is perfectly normal, $x_{1}$ and $x_{2}$ are contained in a closed subset of a neighborhood $V$ of $x_{1}$ or $x_{2}$ which has the property that there exist numbers $a_{1}$ and $a_{2}$ such that if $z \in V, \alpha_{p}(z)>a_{1}>g\left(x_{1}\right)>a_{2}>\beta_{p}(z)$. If there is a neighborhood of $x_{1}$ which does not contain $x_{2}$ and vice versa, then there exist a neighborhood $V$ of $x_{1}$ and a neighborhood $W$ of $x_{2}$ such that $x_{1}$ belongs to a closed subset of $V$ which does not intersect $W$ and there exist numbers $a_{1}$ and $a_{2}$ such that if $z \in V, \alpha_{p}(z)>a_{1}>g\left(x_{1}\right)>a_{2}>\beta_{p}(z)$. The neighborhood $W$ has similar properties. In either case Urysohn's lemma can be used to modify $g_{p}$ such that it agrees with $g$ at $x_{1}$ and $x_{2}$ but is still continuous and between $\alpha_{p}$ and $\beta_{p}$. This process can be continued for a finite number of points of $T$.

The sequence $\left\{g_{p}\right\}_{p=1}^{\infty}$ converges to $g$. If $x \in R, f(x)=g(x)$.

(c) $3 \rightarrow 1$ : Suppose $\left\{g_{p}\right\}_{p=1}^{\infty}, g_{p} \in C(S)$, converges to $g$. Denote the members of $T$ as $\left\{x_{1}, x_{2}, \cdots\right\}$. For each positive integer $p$ define 


$$
\begin{aligned}
f_{p}(x) & =f(x) \quad \text { if } x=x_{n}, & & n \leqq p, \\
& =g_{p}(x) \text { if } x \neq x_{n}, & & n \leqq p,
\end{aligned}
$$

for $x \in S$. The function $f_{p}$ has at most a finite number of discontinuities, each of which is removable. The sequence $\left\{f_{p}\right\}_{p=1}^{\infty}$ converges to $f$.

\section{REFERENCES}

1. F. Hausdorff, Set theory, 2nd. ed., Chelsea, New York, 1962.

2. Hing Tong, Some characterizations of normal and perfectly normal spaces, Duke Math. J. 19 (1952), 289-292.

3. K. Nagami, Baire sets, Borel sets and some typical semi-continuous functions, Nagoya Math. J. 7 (1954), 85-93.

UNIVERSITY OF HOUSTON 